\title{
A Situation Analysis Decision Support System Based on Dynamic Object Oriented Bayesian Networks
}

\author{
Mohsen Naderpour and Jie Lu \\ Decision Systems and e-Service Intelligence Laboratory \\ Centre for Quantum Computation \& Intelligent Systems, School of Software \\ Faculty of Engineering and IT, University of Technology, Sydney \\ Mohsen.Naderpour@student.uts.edu.au, Jie.Lu@uts.edu.au
}

\begin{abstract}
This paper proposes a situation analysis decision support system (SADSS) for safety of safety-critical systems where the operators are stressed by the task of understanding what is going on in the situation. The proposed SADSS is developed based on a new model-driven engineering approach for hazardous situations modeling based on dynamic object oriented Bayesian networks to reduce the complexity of the decision-making process by aiding operators' cognitive activities. The SADSS includes four major elements: a situation data collection based on observable variables such as sensors, a situation knowledgebase which consists of dynamic object oriented Bayesian networks to model hazardous situations, a situation analysis which shows the current state of hazardous situations based on risk concept and possible near future state, and a humancomputer interface. Finally two evaluation methods for partial and full validation of SADSS are presented.
\end{abstract}

Index Terms - Situation awareness, decision support systems, safety-critical systems

\section{INTRODUCTION}

In many safety-critical systems, operational safety is the top priority because the consequence of every accident can result in loss of life, environmental pollution, and financial losses. The majority of accidents in these environments are caused by human error. For instance, a review showed that the human error contribution in air traffic management accidents is more than $90 \%$, and most industries have similar human error contributions i.e. 70 $90 \%$ [1]. Chernobyl disaster in 1986 is a famous event that was caused by human errors that dealt with decision making, receiving information, and action selection. Therefore to maintain safety in such complex systems, human factor issues cannot be ignored.

Later, the human factors researches showed that in many of accidents, operators were struggling against significant challenges. They have to face both data overload and the challenge of working with a complex system. In fact, the persons are not the cause of these errors but they have inherited the problems and

Manuscript received September 5, 2013; revised September 25, 2013; accepted October 5, 2013. difficulties from the technologies that engineers have created. Operators generally have no difficulty in physically performing their tasks, and no difficulty in knowing what is the correct thing to do, but they are stressed by the task of understanding what is going on in the situation [2]. Over the last two decades, great deal of research has been undertaken in the area of situation awareness (SAW). Situation awareness, a state in the mind of human, is essential to conduct decision-making activities. It is about the perception of the elements in the environment, the comprehension of their meaning, and the projection of their status in the near future [2]. Situation analysis (SA) is defined as a process, the examination of a situation, its elements, and their relations, to provide and maintain a product, i.e. SAW. One important aspect of SA is to focus attention to relevant situations. When a decision maker is aware of the current situation and its future implications it can be quite straight forward to decide on which action to take. After an action is performed, the cycle is completed by once again observing the universe of discourse and analyzing if the chosen action had the desired effect. This iterative process is perhaps better known as the OODA (Observe, Orient, Decide and Act) loop. The OODA loop is a cyclic process that allows for concepts such as SA and SAW to be related to decision making as shown in Fig. 1 [3].

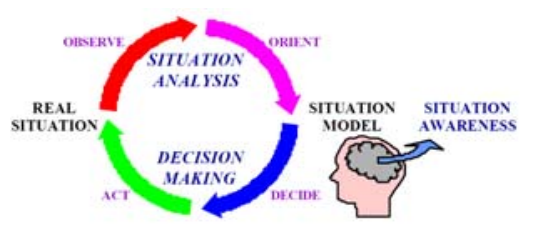

Figure 1. Situation analysis and decision making.

In a technological system, the operators' tasks include information gathering, planning, decision making, and avoiding unforeseen risks through the operation system. They monitor the system continually to ensure that the system is stable and functioning normally. During abnormal situations, a well-trained operator should comprehend a malfunction in real time by analyzing alarms, assessing values, or recognizing unusual trends of 
multiple instruments. Today in a complex system, many alarms from many different systems often occur at the same time during an incident, making it difficult for the operator to select a correct response efficiently. In the absence of operator support systems, the operators must consider an overwhelming amount of information and make decisions very quickly. Since the decision-making environment is extremely complicated, the use of automated systems to aid decision making is highly recommended. This paper focuses on the development of a decision support system to improve operators' SAW and to lower the chance of human errors.

\section{BACKGROUND}

\section{A. Situation Awareness}

One of the earliest and most widely used definitions of SAW describes it as the "perception of the elements in the environment within a volume of time and space, the comprehension of their meaning and the projection of their status in the near future" [2]. Based on this definition, SAW includes three levels: 1) perception, 2) comprehension, and 3) projection.

Level 1 SAW, perception, involves the sensory detection of significant environmental cues. For example, operators need to be able to see relevant displays or hear an alarm sound. Comprehending the meaning or significance of that information in relation to one's goals is also important. Operators with good Level 2 SAW are able to understand the immediate impact of an outage on other parts of the system. Projection, the highest level of SAW, consists of extrapolating information forward in time to determine how it will affect future states of the operating environment. This merges what the individual knows about the current situation with their mental models of the system to predict what is likely to happen next. The higher levels of SAW allow operators to function in a timely and effective manner, even with very complex and challenging tasks.

\section{B. Bayesian Networks Theory}

A BN is defined as a couple: $\mathcal{G}=((N, A), \mathcal{P})$, where $(N, A)$ represents the graph; $N$ is a set of nodes; $A$ is a set of arcs; $\mathcal{P}$ represents the set of probability distributions that are associated to each node. When a node is not a root node, the distribution is a conditional probability distribution that quantifies the probabilistic dependency between that node and its parents. A discrete random variable $X$ is represented by a node $n \in N$ with a finite number of mutually exclusive states. States are defined on $S_{n}:\left\{s_{1}^{n}, s_{2}^{n}, \ldots, s_{M}^{n}\right\}$. The set $\mathcal{P}$ is represented with Conditional Probability Tables (CPT). Then, each node has an associated CPT. For instance, the nodes $n_{i}$ and $n_{j}$ are defined over the sets $S_{n_{i}}:\left\{s_{1}^{n_{i}}, s_{2}^{n_{i}}, \ldots, s_{M}^{n_{i}}\right\}$ and $S_{n_{j}}:\left\{s_{1}^{n_{j}}, s_{2}^{n_{j}}, \ldots, s_{L}^{n_{j}}\right\}$. The CPT of $n_{j}$ is then defined by the conditional probabilities $p\left(n_{j} \mid n_{i}\right)$ over each $n_{j}$ state knowing its parents states $\left(n_{i}\right)$. This CPT is defined as a matrix:
$P\left(n_{j} \mid p a\left(n_{j}\right)\right)=$

$\left[\begin{array}{ccc}p\left(n_{j}=s_{1}^{n_{j}} \mid n_{i}=s_{1}^{n_{i}}\right) & \ldots & p\left(n_{j}=s_{L}^{n_{j}} \mid n_{i}=s_{1}^{n_{i}}\right) \\ \vdots & \vdots & \vdots \\ p\left(n_{j}=s_{1}^{n_{j}} \mid n_{i}=s_{M}^{n_{i}}\right) & \cdots & p\left(n_{j}=s_{L}^{n_{j}} \mid n_{i}=s_{M}^{n_{i}}\right)\end{array}\right]$

Various inference algorithms can be used to compute marginal probabilities for each unobserved node given information on the states of a set of observed nodes. The most classical one relies on the use of a junction tree. Inference in $\mathrm{BN}$ then allows to take into account any state variable observation (an event) so as to update the probabilities of the other variables. When observations are given, this knowledge is integrated into the network and all the probabilities are updated accordingly.

A hard evidence of the random variable $X$ indicates that the state of the node $n \in N$ is one of the states $S_{n}:\left\{s_{1}^{n}, s_{2}^{n}, \ldots, s_{M}^{n}\right\}$. Nevertheless, when this knowledge is uncertain, soft evidences can be used. A soft evidence for a node $n$ is defined as one that enables the updating of the prior probability values for the states of $n$.

A dynamic BN (DBN) is a BN that includes a temporal dimension. This new dimension is managed by timeindexed random variables $X_{i}$ is represented at time step $k$ by a node $n_{(i, k)} \in N$ with a finite number of states $S_{n_{i}}:\left\{s_{1}^{n_{i}}, s_{2}^{n_{i}}, \ldots, s_{M}^{n_{i}}\right\}$. Several time stages are represented by several sets of nodes and an arc that links two variables belonging to different time slices represents a temporal probabilistic dependence between these variables. Then DBNs allow to model random variables and their impacts on the future distribution of other variables. Defining these impacts as transition probabilities between the states of the variable at time step $k-1$ and those at time step $k$ leads to the definition of CPTs, that are relative to inter-time slices. With this model, the future slice $(k)$ is conditionally independent of the past given the present $(k-1)$ [4].

Modeling systems containing an important number of variables with BNs generally leads to complex models. To avoid this phenomenon, a particular class of BNs, the Object Oriented Bayesian Networks (OOBNs) has been defined [5]. This modeling is based on the decomposition of the global network into hierarchical levels. This representation method allows to decentralize and to structure the knowledge within BNs of reduced size. An OOBN class is a BN fragment containing output, input, and protected (or encapsulated) nodes. The input and output variables form the interface of the class. The interface encapsulates the internal variables of the class, d-separating them from the rest of the network. All communication with other instances is formulated in terms of probability statements over the instance's interface.

\section{Fuzzy Sets}

Fuzzy logic deals with problems that have vagueness, uncertainty, or imprecision, and uses membership functions with values varying between 0 and 1. In fuzzy set theory based on fuzzy logic, a particular object has a degree of membership in a given set that may be anywhere in the range of 0 to 1 . Fuzzy theory was first 
proposed by Zadeh in 1965. A fuzzy logic system (FLS) consists of three parts: fuzzification of the input variables, fuzzy inference process and defuzzification. In the fuzzification process, the fuzzy sets are formed for all variables. The fuzzy inference engine takes into account the input variables and the logic relations between them and uses fuzzy logic operations to generate the output. Mamdani's fuzzy inference method is the most commonly seen fuzzy methodology. This inference includes the implication in each single rule and the aggregation from all rules. All output functions returned by the implication process for each rule are combined into a single output fuzzy set. In the defuzzification stage, the crisp value of output is obtained using a centroid method [6].

\section{A Situation ANALysis Decision Support System}

Decision making, by its nature, is a cognitive process, involving different cognitive tasks, such as collecting information, evaluating situation, generating and selecting alternatives, and implementing solutions. Decision making is never error-proof, as decision makers are prone to their cognitive biases. Therefore, decision support systems (DSSs) are often used by decision makers in order to minimize their cognitive errors and maximize the performance of actions. Based on the model of assistance, DSS can be categorized into various types, such as model-driven DSS, communication-driven DSS, data-driven DSS, document-driven DSS and knowledge-driven DSS [7].

In this paper we propose a situation analysis decision support system (SADSS) which is a model-driven DSS as shown in Fig. 2. The model-driven DSSs include computerized systems that use the quantitative models to assist in decision-making. In fact, the model-driven DSSs emphasize access to and manipulation of the quantitative models and hence the models are the dominant component in the DSS architecture that provide the functionality for the DSS [8]. The proposed system consists of four major components: 1) situation data collection (e.g. observable variables such as sensors), 2) situation knowledge base, 3) situation analysis, and 4) human-computer interface.

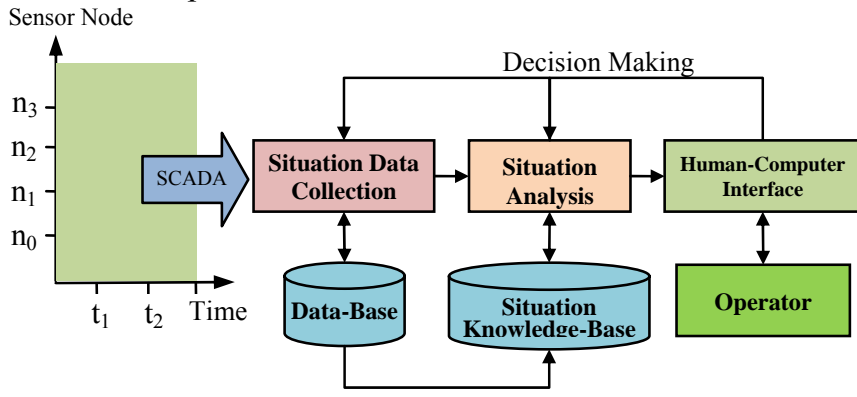

Figure 2. A situation analysis decision support system.

\section{A. The SAW Requirements}

To determine the aspects of a situation that are important for a particular user's SAW, we use a methodology which is called the goal-directed task analysis (GDTA). This methodology is a specific form of cognitive task analysis that focuses on identifying the goals and critical information needs for a task context. The GDTA process forms an exemplary template for incorporating human cognition into an actionable model by describing in detail not only a user's information data needs (Level 1), but also how that information needs to be combined to form the comprehension (Level 2), and projection of future events (Level 3) that are critical to SAW, thereby providing a critical link between data input and the decisions to be made in a goal-directed environment [2]. In this paper, the elements of GDTA include goal, subgoals, decisions and SAW requirements are determined as shown in Table I. The main goal of SADSS, elimination of the risk to a level that is as low as reasonably practicable (ALARP), is followed by two subgoals: risk determination and risk reduction. Associated with each subgoal, the major decisions that need to be made are then identified. The SAW needed for making these decisions and carrying out each sub-goal are determined.

TABLE I.

SAFETY GOALS, DECISIONS AND SAW REQUIREMENTS

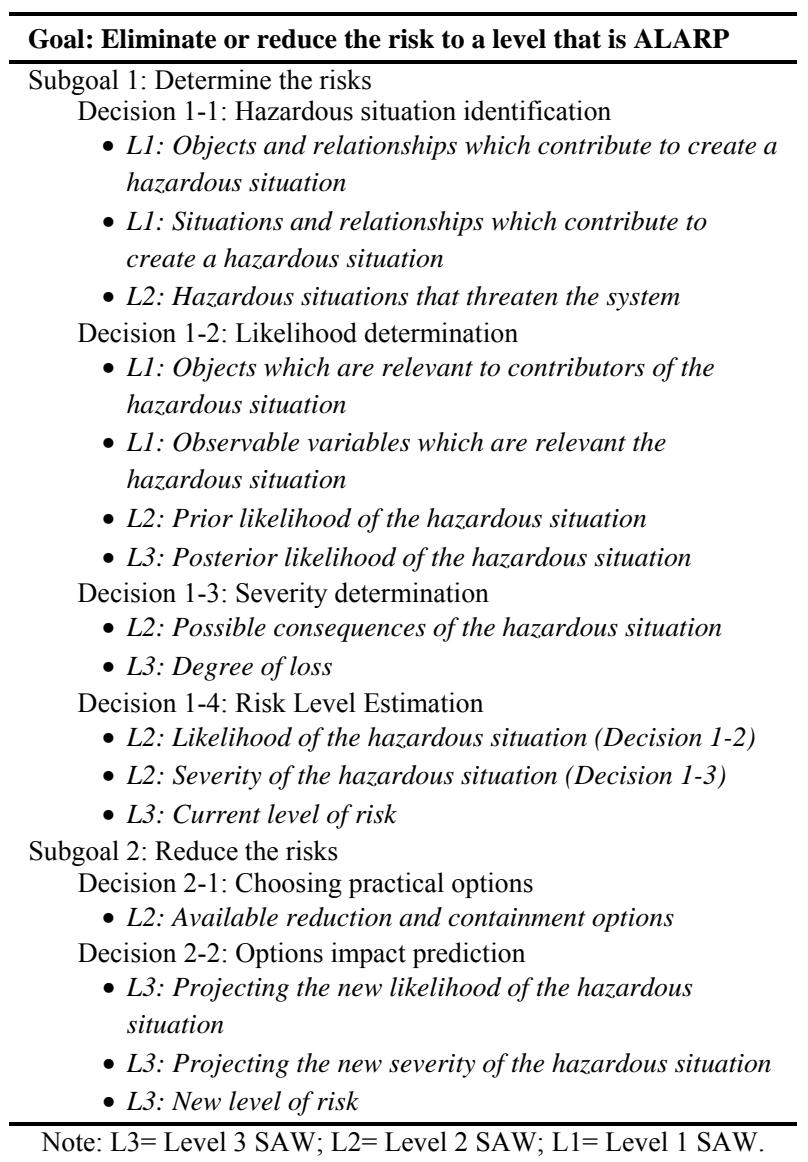

\section{B. Constructing the Situation Knowledge-Base}

Bayesian network is a kind of cause and effect inference method under uncertainty with directed acyclic graphical mode that encodes the probability relationship. However, one of the main obstacles is to create and maintain very large domain models. To remedy this problem, the OOBNs have been proposed to solve the 
represent method of the complex system. The module BN can decompose the complex problem into simple subproblem. Then the BN model for each sub-problem is built and linked into a whole BN model according to the relation between the sub-problems lastly. In this paper, to model the hazardous situations, the dynamic OOBNs are relied according to below definitions.

Definition 1 (Situation): A situation is a collection of physical or conceptual objects which have relationships to each other and the environment.

In the object oriented paradigm the basic component is the object; an entity with identity, state and behaviour.

Definition 2 (Hazardous situation): A hazardous situation is defined as a circumstance immediately before harm is produced by a hazard.

Definition 3 (First level hazardous situation): A situation is defined as a first level hazardous situation, if the interactions among its objects create a hazard.

For instance, high temperature inside a mixing tank is a first level hazardous situation because it is produced by interaction of its objects which are a sensor, a pneumatic unit, and a manual steam valve.

Definition 4 (Higher level hazardous situation): A situation is defined as a higher level hazardous situation, if the relationships among other situations and the environment create it.

In the above example, accumulated vapor at the mixing tank environment is a higher level hazardous situation because it is created by two other hazardous situations, high temperature inside the tank and inadequate ventilation in the production unit.

The first level situations can be illustrated by a simple $\mathrm{BN}$, based on its objects. Usually the $\mathrm{BN}$ begins with root nodes that include the basic objects, and follows by a pivot node and leaf nodes. The pivot node is the focal object which delegates the situation. The leaf nodes may be safety barriers which are physical objects of the environment and will connect to each other if there is relation between their performances. Also, one of the leaf nodes may be a consequence node which has some states, and shows the possible accidents, that the situation may create.

According to OOBN, a class is a $\mathrm{BN}$ fragment containing three sets of nodes [5]:

- $O$ is a set of output nodes. Output nodes can be referenced outside the class, hence they can be parents of nodes outside instances of the class.

- $I$ is a set of input nodes. Input nodes represent nodes that are actually not in the class; they act as place-holders for parents of nodes inside instances of the class. Input nodes cannot have parents within the class.

- $P$ is a set of protected nodes, i.e. nodes that can only have parents and children inside the class itself.

A class encapsulates nodes and restricts the visibility of its nodes to the interior, in order to use a class it must be instantiated. When an instantiation of a class is created, it can be linked to the rest of the network by reference link. In this paper we use the class definition to develop similar situations. For instance, in the above example, if the environment includes 5 similar mixing tanks, then 5 instances of the high temperature situation class can be created. This enables us to overcome the challenges of working with traditional BN.

The higher level hazardous situations can be inferred from other situations. Several situations can exist in parallel or the existence of one situation can exclude the existence of another situation. Additionally the class definition can be used for higher level hazardous situations modeling. There are also situations which can only be inferred by observing the real world over a period of time. Although the situations are characterized by information collected over a time-period, they only exist at a special point in time. Their existence in the next time-point has to be verified again. The complete modeling of the dependencies results a knowledge-base of hazardous situations.

\section{Situation Data Collection}

The situation data collection provides the current state of the observable variables based on the online condition and process monitoring systems. According to the conditions and process monitoring, each observable variable value is obtained from field sensors based on $\mathrm{SCADA}^{1}$ systems. The component stores these data in a database where they will be used to modify the CPTs of $\mathrm{BN}$ models in the future. Additionally, the component conducts a discretization process for continuous observable variables to be prepared as soft evidence in order to use in the subsequent situation analysis component. In general, mapping a continuous variable to a discrete variable can be done with a crisp set or a fuzzy set. The literature shows that the concept of fuzzy set theory can provide a smoother and structured way to improve the classical discretization techniques. Therefore in this paper, to discretize the continuous variables, a fuzzy partitioning method is used according to below definitions.

Definition 5 (Fuzzy partition): A fuzzy partition on the universe $\Omega$ is a set of fuzzy sets $\left\{q_{1}, q_{2}, \ldots, q_{m}\right\}$ such that:

$$
\forall x \in \Omega, \sum_{i=1}^{m} \mu_{q_{i}}(x)=1
$$

where $\mu_{q_{i}}(x)$ is the membership function of $q_{i}$.

Definition 6 (Fuzzy state): Let $\left\{q_{1}, q_{2}, \ldots, q_{m}\right\}$ be a fuzzy partition on the universe $\Omega$, then every fuzzy set $q_{i}$, $i=1, \ldots, m$ is defined as a fuzzy state such that:

$$
q_{i}=\left\{\mu_{q_{i}}(x) \mid x \in \Omega\right\}
$$

To simplify the inference process for a continues variable $X_{i}$, consider the fuzzy partition $\left\{q_{1}, q_{2}, \ldots, q_{m}\right\}$. Define $H_{j}(j=1,2, \ldots, m)$ as hypotheses that $X_{i}$ is in fuzzy state $q_{j}$. The results of membership functions $\mu_{q_{j}}(x)$ $j=1,2, \ldots, m$ form the soft evidence vector:

$$
e=\left\{\mu_{q_{1}}(x), \mu_{q_{2}}(x), \ldots, \mu_{q_{m}}(x)\right\}
$$

\footnotetext{
Supervisory Control and Data Acquisition
} 
The $\mu_{q_{j}}(x)$ is considered to be approximately equivalent to the condition probability $P\left(q_{j} \mid X_{i}=x\right)$ [9].

\section{Situation Analysis}

Based on goals and the scope of the operator's responsibility, the situations of interest are recalled from situation knowledge-base. The $\mathrm{BN}$ models provide a graphical situational network for operator. According to the situation models, the probabilities of the existence of the first level hazardous situations are inferred directly from their objects, and the probabilities of the higher level hazardous situations are calculated based on the existence probability of other situations. This also includes temporal dependencies, i.e. that the existence probability of an inferred situation in future can be supported by the earlier existence of the situation itself. Based on the situational network, the prior and posterior probability of the situations are available at any time. The situation analysis component generates an estimation level of risk for every situation to show the operator that the current risk level is acceptable or not. As such estimation is highly subjective and related to inexact information, the application of fuzzy set theory is appropriate. Generally, the risk model calculation follows a multi-step process:

- Estimation of the situation likelihood (i.e. Decision 1-2)

- Estimation of the situation severity (i.e. Decision 1-3)

- Estimation of the situation risk (i.e. Decision 1-4)

To determine the prior and posterior likelihood, the situational network provides the required quantities. The quantitative analysis can proceed along two lines, the forward (or predictive) analysis and backward (or diagnostic) analysis. In forward analysis, the probability of occurrence of any situation of the network is calculated on the basis of the prior probabilities of the objects and the conditional dependence of each node, and backward analysis consists of computing the posterior probability distribution of any situation and object, given the observation of a set of evidence variables.

Usually, the occurrence of a hazardous situation may lead to a broad range of consequences, some of which will probabilistically be undesirable events. Generally, the loss of a consequence may be categorized into one of four groups; asset loss, human fatality, environmental loss, and confidence or reputation loss or a combination thereof. It is useful for all four components to be converted in a common currency such as money for potential comparison and aggregation. Table II shows the proposed severity matrix of this paper.

To estimate the risk level of every situation, a FLS is used. The selection of the membership functions depends on the variable characteristics, available information and expert knowledge. In this paper, the shapes of the membership functions are defined as a combination of trapezoidal and triangular numbers to simplify the operation and increase the sensitivity in some bounds. Fig. 3 illustrates the proposed fuzzy sets. The fuzzy inference engine takes into account the input variables, likelihood and severity, and the logic relations between them including the 25 rules as shown in Table III, and uses fuzzy logic operations to generate the output i.e. risk.

TABLE II:

CONSEQUENCE SEVERITY MATRIX.

\begin{tabular}{ccccc}
\hline $\begin{array}{c}\text { Severity } \\
\text { Class }\end{array}$ & $\begin{array}{c}\text { Monetary } \\
\text { Value }\end{array}$ & $\begin{array}{c}\text { Human } \\
\text { Loss }\end{array}$ & Asset Loss & Environment Loss \\
\hline Negligible & $<10 \mathrm{k}$ & $\begin{array}{c}\text { One minor } \\
\text { injury }\end{array}$ & $\begin{array}{c}\text { Minor repairs that } \\
\text { can be done } \\
\text { immediately by own } \\
\text { crew }\end{array}$ & $\begin{array}{c}\text { Around the area, } \\
\text { easy recovery }\end{array}$ \\
\hline Minor & $10-100 \mathrm{k}$ & $\begin{array}{c}\text { One or two } \\
\text { minor } \\
\text { injuries }\end{array}$ & $\begin{array}{c}\text { Repairs that take } \\
\text { several days to carry } \\
\text { out }\end{array}$ & $\begin{array}{c}\text { Within plant, short } \\
\text { term remediation } \\
\text { effort }\end{array}$ \\
\hline Medium & $100 \mathrm{k}-$ & $\begin{array}{c}\text { Multiple } \\
\text { major } \\
\text { injuries }\end{array}$ & $\begin{array}{c}\text { Damage that takes } \\
\text { months to repair and } \\
\text { causes serious } \\
\text { consequences }\end{array}$ & $\begin{array}{c}\text { Minor offsite impact, } \\
\text { remediation cost will } \\
\text { be less than 1 million }\end{array}$ \\
Major & $\begin{array}{c}1-10 \\
\text { million }\end{array}$ & $\begin{array}{c}\text { One fatality } \\
\text { or multiple } \\
\text { injuries } \\
\text { with } \\
\text { disabilities }\end{array}$ & $\begin{array}{c}\text { Very large material } \\
\text { damage }\end{array}$ & $\begin{array}{c}\text { Community advisory } \\
\text { issued, remediation } \\
\text { cost remains below } \\
10 \text { million }\end{array}$ \\
\hline Catastrophic $>10$ million & $\begin{array}{c}\text { Multiple } \\
\text { fatalities }\end{array}$ & $\begin{array}{c}\text { Significant parts of } \\
\text { the system } \\
\text { destroyed }\end{array}$ & $\begin{array}{c}\text { Community } \\
\text { period, remediation } \\
\text { cost in excess of 10 } \\
\text { million }\end{array}$ \\
\hline
\end{tabular}
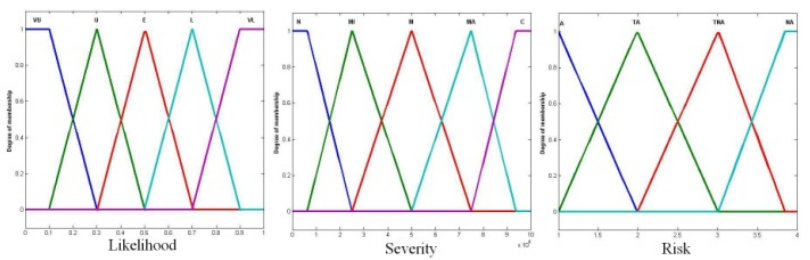

Figure 3. Membership functions of the FLS variables.

TABLE III:

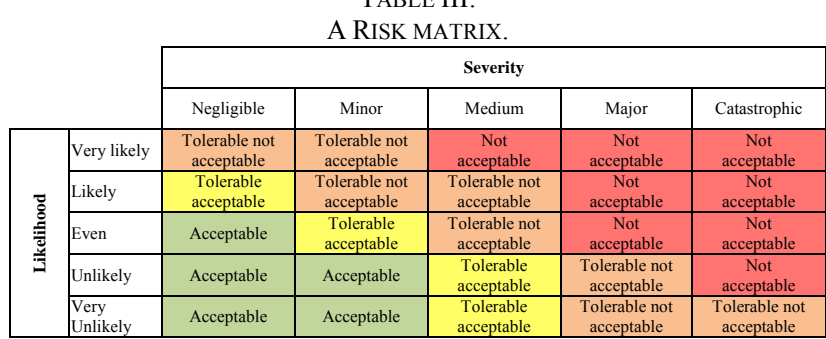

If the estimated risk of the situation is unacceptable, it is necessary to recover the situation. However the proposed situational network does not provide the risk reduction measures, it helps to simulate the impact of risk recovery decisions on the situations. A list of available reduction and containment options can be presented as decision rules to remove or eliminate the risk and recover the situations (i.e. Decision 2-1). Based on the operator's decision to choose the practical options, the situation analysis component has the ability to simulate the situations and estimate the new risk level of situations (i.e. Decision 2-2). The aim is to eliminate or reduce the risk level of potential situations to an acceptable level.

\section{E. Human-Computer Interface}

A graphical user interface (GUI) for the proposed system is being developed based on SAW-oriented design principles and using SMILE (Structural Modeling, Inference, and Learning Engine), which is a library of 
$\mathrm{C}++$ classes for implementing BNs in intelligent systems. The SAW-oriented design principles include general guidelines for supporting SAW, coping with automation and complexity, designing of alarm systems, presenting the information uncertainty, and supporting SAW in team operations. The principles have been used successfully as a design philosophy for systems involving operations, medical systems, flexible manufacturing cells, and command and control for distributed teams [2].

\section{EVALUATION}

Evaluation is an important aspect of every methodology as it provides a reasonable amount of confidence to the results of the model. As in this paper the SADSS was proposed based on DOOBNs, the evaluation of the proposed system can be conducted via two ways: a SAW measurement or a sensitivity analysis. The SAW measurement can be used for a full validation of human-computer interface and the sensitivity analysis is appropriate for partial evaluation of BN models.

\section{A. Situation Awareness Measurement}

The enhancement of SAW is a major design goal for developers of interfaces, automation concepts, and training programs in a verity of fields. To evaluate the degree to which new technologies actually improve operator's SAW, it is necessary to systematically evaluate them based on a measure of SAW, which can determine those ideas that have merit and those that have unforeseen negative consequences. A recent review identified over thirty different approaches designed specifically for the measurement of SAW that can be categorized into the different types: 1) freeze probe techniques, 2) real-time probe techniques, 3) self-rating techniques, 4) observer rating techniques, 5) observer rating techniques, 6) performance measures, and 7) process indices. However, the literature showed that the SAGAT ${ }^{1}$ which is a freeze probe technique, and the $\mathrm{SART}^{2}$ which is a self-rating approach, are by far the most commonly applied during individual and team SAW assessments [10].

\section{B. Sensitivity Analysis}

As in this paper to develop the situation knowledgebase, DOOBNs were relied, therefore a partial validation can be investigated by a sensitivity analysis according to the following three axioms [11]:

- A slight increase/decrease in the prior subjective probabilities of each parent node should certainly result in the effect of a relative increase/decrease of the posterior probabilities of the child node.

- Given the variation of subjective probability distributions of each parent node, its influence magnitude to the child node values should keep consistency.

- The total influence magnitudes of the combination of the probability variations from $x$ attributes (evidence) on the values should be always greater

\footnotetext{
${ }^{1}$ Situation Awareness Global Assessment Technique

2 Situation Awareness Rating Technique
}

than the one from the set of $x-y(y \in x)$ attributes (sub-evidence).

\section{CONCLUSION AND FUTURE WORK}

This paper has presented a model-driven SADSS based on the identified SAW requirements, DOOBNs and fuzzy risk analysis concept. The proposed SADSS includes four major elements. First, it consists of a situation data collection component, which provides the new information of currents situations from SCADA monitoring system. Second, it includes a situation knowledge-base based on DOOBNs. Third, the situation analysis based on current $\mathrm{BN}$ models provides the risk level of situations to show the operator that the current risk level is acceptable or not. Also it can show the possible future state of every situation. Fourth, the SADSS enjoys a GUI. Situation analysis processes are often distributed in nature and involve multiple operation operators that observe and react to events distributed in time and space, so the future direction of the research is to extend the proposed system to a distributed system that applies a team situation awareness concept.

\section{REFERENCES}

[1] A. Isaac, S. T. Shorrock, and B. Kirwan, "Human error in European air traffic management: the HERA project," Reliability Engineering \& System Safety, vol. 75, pp. 257$272,2002$.

[2] M. R. Endsley, "Situation awareness," in Handbook of Human Factors and Ergonomics, G. Salvendy, Ed.: John Wiley and Sons, 2006, pp. 528-542.

[3] J. Roy, "From data fusion to situation analysis," in The 4th International Conference on Information Fusion, Montreal, Canada, 2001.

[4] P. Weber and L. Jouffe, "Complex system reliability modelling with Dynamic Object Oriented Bayesian Networks," Reliability Engineering \& System Safety, vol. 91, pp. 149-162, 2006.

[5] O. Bangso and P. Wuillemin, "Top-down specification and compact representation of repetitive structures in Bayesian networks," in The 13th International Florida Artificial Intelligence Research Symposium Conference, Orlando, USA, 2000.

[6] M. Naderpour and J. Lu, "A Hybrid Bayesian Network for Safety of Chemical Plants," in The $17^{\text {th }}$ Pacific Asia Conference on Information Systems (PACIS), Jeju Island, Korea, 2013.

[7] L. Niu, J. Lu, and G. Zhang, Cognition-Driven Decision Support for Business Intelligence: Models, Techniques, Systems and Applications vol. 238. Berlin Heidelberg: Springer-Verlag, 2009.

[8] D. J. Power and R. Sharda, "Model-driven decision support systems: Concepts and research directions," Decision Support Systems, vol. 43, pp. 1044-1061, 2007.

[9] M. Naderpour, J. Lu, and G. Zhang, "A Fuzzy Dynamic Bayesian Network-Based Situation Assessment Approach," in The $22^{\text {th }}$ IEEE International Conference on Fuzzy Systems (FUZZ-IEEE), Hyderabad, India, 2013.

[10] N. Stanton, Human Factors Methods: A Practical Guide for Engineering and Design. Aldershot, UK: Ashgate, 2005.

[11] B. Jones, I. Jenkinson, Z. Yang, and J. Wang, "The use of Bayesian network modelling for maintenance planning in a manufacturing industry," Reliability Engineering \& System Safety, vol. 95, pp. 267-277, 2010. 